\title{
A conservative approach for subsolid lung nodules: is it safe enough?
}

\author{
Maurizio Infante
}

Affiliation: Dept of Thoracic Surgery, Humanitas Research Hospital, Milan, Italy.

Correspondence: Dept of Thoracic Surgery, Humanitas Research Hospital, Via Manzoni 56, 20089 Rozzano, Milan, Italy. E-mail: maurizio.infantedhumanitas.it

0 $@$ ERSpublications

A long-term follow-up strategy may be reasonable for subsolid nodules at low risk of progression based on CT features http://ow.ly/HasGF

With the foreseen dissemination of lung cancer screening with low-dose spiral computed tomography (LDCT), a great number of subjects will come to medical attention harbouring small, indeterminate pulmonary nodules [1]. Subsolid lung nodules (SSNs), also called ground-glass opacities or ground-glass nodules (GGNs), are identified in $2-3 \%$ of subjects receiving a screening LDCT $[2,3]$. Many of them have a benign aetiology and resolve spontaneously over time. However, unlike solid nodules that represent true lung cancer in a tiny fraction of patients, nonresolving SSNs are found to be histologically malignant in $30-70 \%$ of the cases [4, 5]. For this reason, they are often considered for immediate resection. Nonetheless, recent reports $[6,7]$ suggest that long-term observation of these patients may be appropriate.

In this issue of the European Respiratory Journal (ERJ), the clinical course of 108 patients with persistent SSNs detected in the NELSON trial is reported [8], and a long-term follow-up approach is proposed on the grounds that the risk of progression of SSNs towards clinically significant lung cancer is extremely low. In principle, despite the high probability of SSNs being histologically malignant, their clinical behaviour tends to be indolent and surgical resection of all of them would therefore lead to a great number of lung resections with little real benefit, i.e. to overdiagnosis and overtreatment. However, given that progression may actually occur over time, a management plan should be devised and discussed on a case by case basis.

Since the mid-1990s, when the association of subsolid nodules with early-stage bronchioloalveolar carcinoma was first reported [9, 10], numerous publications have addressed the clinical significance of SSNs and their relationship with the spectrum of lung adenocarcinoma [11]. A good correlation between the aspect of SSNs on computed tomography (CT), their histological features of malignancy and clinical aggressiveness was observed in Japan a long time ago $[12,13]$ and still has practical value today, although the pathology terms have been changed for the spectrum of lung adenocarcinoma.

Pure GGNs of $<10 \mathrm{~mm}$ are most likely benign atypical adenomatous hyperplasia or, more rarely, adenocarcinoma in situ (AIS). Pure GGNs measuring 10-30 mm reflect AIS or minimally invasive lepidic adenocarcinoma. A solid component in a GGN often reflects tumour invasion of the interstitium; the larger the solid part on CT, the larger the area of invasion $[14,15]$. The relative and absolute sizes of the solid component are also known to correlate with the probability of nodal metastasis, recurrence after surgery and death. Pure GGNs have virtually no probability of nodal involvement, whereas for part-solid nodules, the risk rises to about $15 \%$, and to $\sim 30 \%$ for solid nodules $[15,16]$.

In a recent report on 145 patients with resected stage I lung adenocarcinoma, a solid component volume of $1500 \mathrm{~mm}^{3}$ (equivalent to a radius of $\sim 7 \mathrm{~mm}$ in a spheroid) and/or a solid component representing $\geqslant 63 \%$ of the whole lesion size were independent risk factors for recurrence and death. Only solid or part-solid lesions recurred in those patients [17].

Received: Dec 022014 | Accepted: Dec 132014

Conflict of interest: None declared.

Copyright OERS 2015 
Such radiological-pathological correlation is prognostically important and allows a management strategy to be devised on those grounds. Lung-sparing surgery or standard lobectomy may be planned based on lesion size, the ratio between solid and hazy areas in CT images, and on positron emission tomography results [18-20]. A 100\% 5-year survival rate is expected for pure SSNs measuring <30 mm even after simple wedge resection, a less-than-adequate treatment for ordinary lung cancer [21].

\section{Risk of progression of SSNs to clinically significant lung cancer and how we can anticipate it}

A potential downside of a long-term surveillance approach for SSNs is that some SSNs may ultimately progress beyond stage I while they are being monitored [22]. While surgical management of all persistent SSNs would result in overtreatment in most cases, it might result in undertreatment in some patients. This said, the likelihood of progression over several years was shown to be quite low for pure GGNs measuring $\leqslant 20 \mathrm{~mm}$ (approximately 12-24\%), although not negligible for part-solid GGNs, for which the likelihood of progression was in the range of $50 \%$ at 5 years $[6,23]$.

Several reports indicate that a history of previous lung cancer, increasing age and smoking history, but especially whole nodule size and the size of its solid component are major risk factors for progression during follow-up [24-27]. Progression can manifest itself as an increase in nodule size, the appearance of a new solid component, an increase in the size of an already existing one or more than one of these; rarely, the nodule may shrink while a solid core appears, reflecting tumour invasion into the stroma or, in some cases, nodule density increases (i.e. the SSN becomes more visible on CT) while its size remains unchanged $[22,28]$.

If it occurs, progression tends to be slow: HASEgAWA et al. [29] reported in 2000 a mean volume doubling time (VDT) of $813 \pm 375$ days for pure nonsolid lesions and of $457 \pm 260$ days for part-solid nodules, whereas it was $149 \pm 125$ days for solid nodules. In a recent report by SoNG et al. [30], part-solid GGNs with a solid core measuring $\leqslant 5 \mathrm{~mm}$ had a median VDT of 1228.5 days (range 935-4600 days), which was significantly longer than those with a solid portion $>5 \mathrm{~mm}$.

In the report by SCHOLTEN et al. [8] in this issue of the ERJ shows that volume and mass doubling times of SSNs tend to be very long, and that a cut-off of $<400$ days would be unable to discriminate malignant from benign SSNs. The performance of volumetric software for subsolid nodules is less than ideal. In fact, SCHOLten et al. [8] resorted to manual determinations to obtain diameter, then volume and, hence, mass measurements, and moreover, one-third of their patients were ultimately referred for further workup based on a visual impression of growth. KIM et al. [31] reported an error margin of $-18 \%$ to $+19 \%$ for automated mass measurements of SSNs, which is comparable with the results of SCHOLTEN et al. [8]. An increase in mass of $<30 \%$ may be a reliable indicator of an indolent lesion so, clearly, reliable automated measurements of SSN volume and mass would be desirable, but it seems that clinically significant progression of SSNs may still be grossly estimated even without volumetric software in a community setting $[25,32,33]$.

\section{How safe is it to defer surgical treatment for patients with SSN?}

As the size of the SSN and of its solid component are related to the risk of nodal metastasis and recurrence, radiological evidence of growth is generally considered sufficient indication for surgical treatment if the patient is in a reasonable condition. However, abrupt tumour dissemination while the patient is being followed is unlikely.

The study by SCHOLTEN et al. [8], 79 patients (78\% of all SSNs) progressed (albeit very slowly in most cases) after a median follow-up of 95 months (range 20-110 months), but only 33 were resected. Five patients had benign nodules, nine had pre-invasive lesions and 19 patients had invasive adenocarcinoma, but in only one of them had it progressed beyond stage I. Altogether, only four (3.7\%) patients died of lung cancer in this subgroup. Similar data have been reported by ChAng et al. [7] and Matsuguma et al. [6].

There are few data to infer for how long a patient may be safely followed in the case of radiological progression. SAWADA et al. [34] did not observe any recurrence or progression beyond stage I in a retrospective study of 113 surgically treated patients with SSNs up to $3 \mathrm{~cm}$ who were followed for a median of 9 months prior to resection (range 3-67 months).

SEOK et al. [35] showed that recurrence rates were no different between patients with SSNs that had changed over 6 months and those that had not changed, although the latter had a larger invasive component, on average. In a recent study [36], similar outcomes were observed for subjects with SSNs and biopsy-proven early adenocarcinoma who elected to be followed instead of undergoing immediate resection. This study was, however, small and biased by selection. 
As in the NELSON trial, a surveillance approach was consistently used for SSNs in the screening population of the MILD trial. After analysing the data, SiLVA et al. [28] reached the conclusion that stable subsolid nodules may be monitored for several years without apparently jeopardizing patients' life expectancy. However, a note of caution is appropriate, as especially in younger patients, there is abundant time for their SSNs to progress and potentially turn into clinically aggressive lung cancer during their life span. The patients in the study by Scholten et al. [8] had a median age of 58 years, relatively young by our standards in western countries. What about their SSNs in 10 years' time?

Lessons learned from prostate cancer screening may, in principle, apply to this subgroup of lung cancer patients as well. Active surveillance of asymptomatic early prostate cancer may be reasonable under certain conditions [37]. Nonetheless, the Scandinavian Prostate Cancer Group Study Number 4 showed that there may be a benefit of radical prostatectomy over watchful waiting in younger men and in those with intermediate-risk prostate cancer [38].

So what can we suggest? Before opting for surveillance or action, we certainly need to examine the SSN and take into account whether the lesion is nonsolid or part-solid, how large the solid component is, and whether it is growing or not. Then, after considering age, comorbidity burden and past history, we need to evaluate the risks of suitable treatment options for that specific patient, remembering that treatment-related morbidity may outweigh the potential benefit in terms of survival in the case of slow-growing tumours.

The pros and cons of obtaining a biopsy should be weighed as well: persistent SSNs should be considered malignant until proven otherwise, but if you would rather not resect on clinical and CT grounds, a percutaneous biopsy may simply put the patient in a state of anxiety and prompt a request for intervention. If you believe that resection or any sort of intervention would instead be appropriate, CT-guided biopsy result may substantiate the indication, or halt it. We will also need to educate our patients regarding their condition and try to evaluate their psychological profile while discussing possible strategies.

In conclusion, although immediate resection may not be appropriate in the majority of such patients, a sensible management plan should be drawn on a case-by-case basis, on clinical grounds [39]. The data of SCHOLTen et al. [8] will help to reduce overdiagnosis and overtreatment of indolent lung cancer in the LDCT screening era.

\section{References}

1 Bach PB, Mirkin JN, Oliver TK, et al. Benefits and harms of CT screening for lung cancer: a systematic review. JAMA 2012; 307: 2418-2429.

2 Horeweg N, van der Aalst CM, Vliegenthart R, et al. Volumetric computed tomography screening for lung cancer: three rounds of the NELSON trial. Eur Respir J 2013; 42: 1659-1667.

3 Henschke CI, Yip R, Yankelevitz DF, et al. Definition of a positive test result in computed tomography screening for lung cancer: a cohort study. Ann Intern Med 2013; 158: 246-252.

4 Henschke CI, Yankelevitz DF, Mirtcheva R, et al. CT screening for lung cancer: frequency and significance of part-solid and nonsolid nodules. AJR 2002; 178: 1053-1057.

5 Kim HY, Shim YM, Lee KS, et al. Persistent pulmonary nodular ground-glass opacity at thin-section CT: histopathologic comparisons. Radiology 2007; 245: 267-275.

6 Matsuguma H, Mori K, Nakahara R, et al. Characteristics of subsolid pulmonary nodules showing growth during follow-up with CT scanning. Chest 2013; 143: 436-443.

7 Chang $\mathrm{B}$, Hwang $\mathrm{JH}$, Choi $\mathrm{YH}$, et al. Natural history of pure ground-glass opacity lung nodules detected by low-dose CT scan. Chest 2012; 143: 172-178.

8 Scholten ET, de Jong PA, de Hoop B, et al. Towards a close computed tomography monitoring approach for screen detected subsolid pulmonary nodules? Eur Respir J 2015; 45: 765-773.

9 Jang HJ, Lee KS, Kwon OJ, et al. Bronchioloalveolar carcinoma: focal area of ground-glass attenuation at thin-section CT as an early sign. Radiology 1996; 199: 485-488.

10 Kushihashi T, Munechika H, Ri K, et al. Bronchioloalveolar adenoma of the lung: CT-pathologic correlation. Radiology 1994; 193: 789-793.

11 Oh JY, Kwon SY, Yoon HI, et al. Clinical significance of a solitary ground-glass opacity (GGO) lesion of the lung detected by chest CT. Lung Cancer 2007; 55: 67-73.

12 Aoki T, Nakata $\mathrm{H}$, Watanabe $\mathrm{H}$, et al. Evolution of peripheral lung adenocarcinomas: CT findings correlated with histology and tumor doubling time. AJR 2000; 174: 763-768.

13 Suzuki K, Kusumoto M, Watanabe S, et al. Radiologic classification of small adenocarcinoma of the lung: radiologic-pathologic correlation and its prognostic impact. Ann Thorac Surg 2006; 81: 413-419.

14 Travis WD, Brambilla E, Noguchi M, et al. International Association for the Study of Lung Cancer/American Thoracic Society/European Respiratory Society international multidisciplinary classification of lung adenocarcinoma. J Thorac Oncol 2011; 6: 244-285.

15 Godoy MC, Truong MT, Sabloff B, et al. Subsolid pulmonary nodule management and lung adenocarcinoma classification: state of the art and future trends. Sem Roentgenol 2013; 48: 295-307.

16 Matsuguma H, Yokoi K, Anraku M, et al. Proportion of ground-glass opacity on high-resolution computed tomography in clinical T1 N0 M0 adenocarcinoma of the lung: a predictor of lymph node metastasis. $J$ Thorac Cardiovasc Surg 2002; 124: 278-284. 
17 Yanagawa M, Tanaka Y, Leung AN, et al. Prognostic importance of volumetric measurements in stage I lung adenocarcinoma. Radiology 2014; 272: 557-567.

18 Kadota K, Colovos C, Suzuki K, et al. FDG-PET SUVmax combined with IASLC/ATS/ERS histologic classification improves the prognostic stratification of patients with stage I lung adenocarcinoma. Ann Surg Oncol 2012; 19: 3598-3605

19 Tsutani Y, Miyata Y, Nakayama H, et al. Prognostic significance of using solid versus whole tumor size on high-resolution computed tomography for predicting pathologic malignant grade of tumors in clinical stage IA lung adenocarcinoma: a multicenter study. J Thorac Cardiovasc Surg 2012; 143: 607-612.

20 Okada M, Nakayama H, Okumura S, et al. Multicenter analysis of high-resolution computed tomography and positron emission tomography/computed tomography findings to choose therapeutic strategies for clinical stage IA lung adenocarcinoma. J Thorac Cardiovasc Surg 2011; 141: 1384-1391.

21 Van Schil PE, Asamura H, Rusch VW, et al. Surgical implications of the new IASLC/ATS/ERS adenocarcinoma classification. Eur Respir J 2012; 39: 478-486.

22 Takashima S, Maruyama Y, Hasegawa M, et al. CT findings and progression of small peripheral lung neoplasms having a replacement growth pattern. AJR 2003; 180: 817-826.

23 Chang B, Hwang JH, Choi $\mathrm{YH}$, et al. Natural history of pure ground-glass opacity lung nodules detected by low-dose CT scan. Chest 2013; 143: 172-178.

24 Kim HS, Lee HJ, Jeon JH, et al. Natural history of ground-glass nodules detected on the chest computed tomography scan after major lung resection. Ann Thorac Surg 2013; 96: 1952-1957.

25 Tamura M, Shimizu Y, Yamamoto T, et al. Predictive value of one-dimensional mean computed tomography value of ground-glass opacity on high-resolution images for the possibility of future change. $J$ Thorac Oncol 2014; 9: 469-472.

26 Kobayashi Y, Sakao Y, Deshpande GA, et al. The association between baseline clinical-radiological characteristics and growth of pulmonary nodules with ground-glass opacity. Lung Cancer 2014; 83: 61-66.

27 Eguchi T, Kondo R, Kawakami S, et al. Computed tomography attenuation predicts the growth of pure ground-glass nodules. Lung Cancer 2014; 84: 242-247.

28 Silva M, Sverzellati N, Manna C, et al. Long-term surveillance of ground-glass nodules: evidence from the MILD trial. J Thorac Oncol 2012; 7: 1541-1546.

29 Hasegawa M, Sone S, Takashima S, et al. Growth rate of small lung cancers detected on mass CT screening. $\mathrm{Br} J$ Radiol 2000; 73: 1252-1259.

30 Song YS, Park CM, Park SJ, et al. Volume and mass doubling times of persistent pulmonary subsolid nodules detected in patients without known malignancy. Radiology 2014; 273: 276-284.

31 Kim H, Park CM, Woo S, et al. Pure and part-solid pulmonary ground-glass nodules: measurement variability of volume and mass in nodules with a solid portion less than or equal to $5 \mathrm{~mm}$. Radiology 2013; 269: 585-593.

32 Lee KH, Goo JM, Park SJ, et al. Correlation between the size of the solid component on thin-section CT and the invasive component on pathology in small lung adenocarcinomas manifesting as ground-glass nodules. $J$ Thorac Oncol 2014; 9: 74-82.

33 Eguchi T, Yoshizawa A, Kawakami S, et al. Tumor size and computed tomography attenuation of pulmonary pure ground-glass nodules are useful for predicting pathological invasiveness. PLoS One 2014; 9: e97867.

34 Sawada S, Komori E, Nogami N, et al. Evaluation of lesions corresponding to ground-glass opacities that were resected after computed tomography follow-up examination. Lung Cancer 2009; 65: 176-179.

35 Seok Y, Cho S, Kim K, et al. Partly solid pulmonary nodules: waiting for change or surgery outright? Interact CardiovascThorac Surg 2014; 19: 556-560.

36 Gulati CM, Schreiner AM, Libby DM, et al. Outcomes of unresected ground-glass nodules with cytology suspicious for adenocarcinoma. J Thorac Oncol 2014; 9: 685-691.

37 Horwich A, Hugosson J, de Reijke T, et al. Prostate cancer: ESMO Consensus Conference Guidelines 2012. Ann Oncol 2013; 24: 1141-1162.

38 Bill-Axelson A, Holmberg L, Garmo H, et al. Radical prostatectomy or watchful waiting in early prostate cancer. N Engl J Med; 370: 932-942.

39 Infante $\mathrm{M}$, Berghmans T, Heuvelmans MA, et al. Slow-growing lung cancer as an emerging entity: from screening to clinical management. Eur Respir J 2013; 42: 1706-1722. 